\title{
Drapeau, M.-J., Sheehan, C.-A., De Celles, A. et Blondin, J. (2020). L'après inc. Vivre sa sortie entrepreneuriale positivement. Septembre éditeur
}

\author{
Daniel Lemay ${ }^{\mathrm{a}}$
}

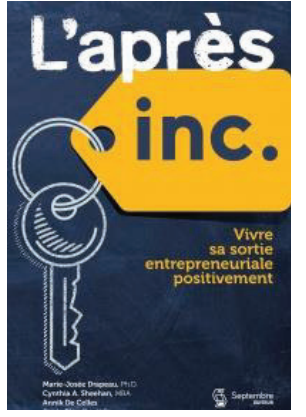

Écrire un livre seul ou en groupe, c'est sans doute un exercice fort exigeant. Du moins, telle est la perception que j'entretiens face à un tel exercice. Il y a une certaine mise à nu; des lecteurs vont aimer, d'autres moins. Selon moi, il s'agit d'un acte empreint d'une certaine humilité. J'ai donc beaucoup de respect pour les gens qui tentent cet exercice, qui constitue un exercice entrepreneurial jusqu'à un certain point. Beaucoup d'efforts y sont consentis et les résultats sont indépendants de la volonté de l'auteur.

J'ai pris connaissance du livre L'après inc. : vivre sa sortie entrepreneuriale positivement, corédigé par Marie-Josée Drapeau, une collègue du DSEA à l'UQAC. Le sujet m’intéressait pour deux raisons. La première : la curiosité. L'entrepreneur est un être toujours en action ayant beaucoup de projets. Or, dans cet ouvrage, on discute plutôt de sa sortie, de son départ, de la fin de sa vie d'entrepreneur. Il s'agit d'une posture non conventionnelle. Deuxièmement, je me suis retrouvé dans cette situation il y a quelques années, donc j'étais curieux de connaitre la façon dont ce sujet pouvait être abordé. Puisque chaque contexte de sortie d'une entreprise est différent, je m’interrogeais sur la possibilité d'aborder ce sujet à l'aide d'une démarche globale.

L'entrepreneur a un projet? Il le crée. Il est dans l'action chaque jour de sa vie d'entrepreneur. Par contre, un jour ou l'autre, il devra faire face à l'inévitable : sa sortie, qu'elle soit souhaitée ou non. C'est l'essence même du livre recensé. Après l'entreprise, la vie est moins rythmée, mais elle continue. Pour s'assurer qu'elle continue d'être belle, certaines étapes doivent être franchies.

Afin de structurer leur démarche, les autrices ont subdivisé le livre en quatre parties séquentielles, chacune identifiée par un verbe d'action : réfléchir, choisir, gérer et rebondir. Selon les autrices, il faut commencer par réfléchir à sa situation d'entrepreneur sortant avant de procéder au choix (choisir). Une fois ce choix effectué, il faut le mettre en œuvre. La section gérer explique cet aspect. Finalement, la partie rebondir informe le lecteur de certaines notions à mettre de l'avant afin de poursuivre sa vie après l'entrepreneuriat, sa transition.

\section{$1^{\text {re }}$ partie : réfléchir}

Les autrices abordent cet aspect en fonction de différents types de signaux apparaissant chez l'entrepreneur, signaux qui expriment une certaine prise de conscience. D'abord, les signaux d'ordre personnel (manque de motivation, problèmes de santé), d'ordre interpersonnel (commentaires de collègues, de la famille, d'amis qui laissent croire qu'il se passe quelque chose) ou encore financiers (difficulté à payer les fournisseurs, baisse de rentabilité, surveillance accrue des institutions financières).

\footnotetext{
${ }^{a}$ Professeur, département des sciences économiques et administratives, Université du Québec à Chicoutimi
} 
L'entrepreneur doit décoder ces signaux, mais comment faire? Les évènements surviennent vite et séparément, mais ils peuvent aussi survenir simultanément. Souvent, l'entrepreneur est pris dans un tourbillon. Il peut alors devenir difficile de prendre le recul nécessaire pour évaluer la conjoncture.

L'ouvrage propose des étapes qui aideront la personne à structurer sa démarche de réflexion. Selon moi, les interrogations abordées sont fondamentales. Elles guideront le lecteur vers les vrais enjeux associés à cette période de turbulence.

\section{$2^{\mathrm{e}}$ partie : choisir}

Dans cette section, les autrices nous présentent différents choix pouvant s'offrir à l'entrepreneur qui prend la décision de se retirer à la suite de sa réflexion. Elles font une analogie avec le football nord-américain, sport que j’affectionne particulièrement.

Dans les trois situations décrites qui réfèrent au football, il s'agit de passer le ballon, de vendre le ballon ou de dégonfler le ballon. Chaque situation fait l'objet d'un chapitre.

La première situation fait référence à la transmission ordonnée de l'entreprise à des membres de sa famille ou à des employés qui voudraient la racheter (passer le ballon). Vendre le ballon réfère à l'action de vendre l'entreprise. La différence entre transférer et vendre, c'est le futur acquéreur : l'entreprise est vendue soit à des personnes connues (famille ou employés), soit à des acheteurs externes non choisis par l'entrepreneur.

La troisième situation, dégonfler le ballon, fait référence à l'arrêt pur et simple de l'activité. Dans les deux dernières situations, le choix de vendre le ballon ou de le dégonfler peut être imposé par la situation dans laquelle se trouve l'entreprise. En général, l'aspect financier peut forcer ce type de décision. D'ailleurs, dans ces situations particulières, l'élément émotionnel associé au fait de se retirer sera amplifié par un sentiment d'échec, qui ajoute à la difficulté à faire face à la situation.

Le transfert de l'entreprise nécessite en premier lieu de trouver un repreneur. L'enjeu est important, car il y a un objectif de pérennité de la société. L'entrepreneur pourra avoir le sentiment de réussite lorsque le transfert sera terminé, mais des étapes importantes doivent être respectées pour qu'il y ait succès. Les autrices identifient les acquéreurs possibles et les enjeux associés à chaque situation, entre autres les enjeux de transmission des pouvoirs, des savoirs et, éventuellement, des avoirs.

Dans la situation où l'entrepreneur vendra son entreprise, les étapes sont différentes. La situation dans laquelle se trouve l'entreprise aura une grande influence sur la facilité avec laquelle cette transaction se conclura par un succès. Un élément essentiel pour maximiser la valeur de la vente est de trouver le bon acheteur - tâche ardue en général.

Dans les situations de transfert ou de vente, il y a un élément financier important. Pour plusieurs entrepreneurs, la disposition de leur entreprise contre des sommes d'argent équivaut à établir le type de retraite qu'ils pourront envisager. En général, un des objectifs de l'entrepreneur lorsqu'il commence son activité est de s'accomplir, mais aussi de s'enrichir et d'assurer une vie confortable pour lui et ses proches. Comme lors de la vente d'une maison, un facteur émotif est associé à la transaction. Il se peut que, pour l'entrepreneur, la valeur perçue de sa compagnie soit plus grande que la valeur perçue par un futur acquéreur. Les autrices sont explicites sur cette perception et un futur vendeur prenant connaissance des informations présentées dans l'ouvrage sera certainement mieux informé et prévenu des pièges «émotifs» qui le guettent. 
Le dernier chapitre de cette section aborde le dégonflement du ballon, soit la fin pure et simple de l'entreprise. Cette fin peut se faire de façon ordonnée, mais peut aussi être exigée par des créanciers. Ce scénario est assurément le pire qu'un entrepreneur puisse expérimenter. En plus de perdre des sommes d'argent importantes, il éprouvera un sentiment d'échec immense. De plus, sa santé pourrait être affectée, du fait que les problèmes financiers ne surviennent pas du jour au lendemain. Il s'agit souvent d'une longue agonie qui laisse des traces.

\section{$3^{\mathrm{e}}$ partie : gérer}

Peu importe le choix effectué, qu’il soit le fruit d’une décision réfléchie ou forcée, il devra être géré. L'entrepreneur subira des impacts directs, mais plusieurs parties prenantes aussi. Il doit réaliser que la vie ne s'arrête pas là et qu'il doit planifier la suite. Plusieurs éléments sont donc à considérer dans la mise en place du choix.

Le premier chapitre de cette partie du livre informe le lecteur sur l'importance de la communication dans le contexte du retrait. On ne communique pas de la même façon s'il y a transfert, vente ou fermeture d'entreprise. Par contre, les parties prenantes sont sensiblement les mêmes : les employés, les clients, les fournisseurs, les institutions financières et les autres partenaires (autres entrepreneurs, amis, relations). Bref, cette étape fait réaliser à l'entrepreneur la quantité de gens qui gravitent autour de son organisation.

Plus importante que tout ce monde, la famille immédiate, qui comprend le conjoint ou la conjointe, les enfants et les parents. En général, l'entrepreneur voit sa famille impliquée indirectement dans son projet. Les longues heures de travail et le stress inévitable sont quelques-uns des impacts vécus par l'entourage immédiat. Les autrices identifient de bonnes pistes qui aideront certainement le lecteur à se poser les bonnes questions. Plusieurs références sont aussi identifiées afin d'aller chercher de l'aide, s'il en ressent le besoin.

La communication d'une décision aussi importante que de se retirer amène des interrogations incontournables. Etre entrepreneur est un statut, et celui-ci disparaitra. Ce passage à vide est abordé dans le deuxième chapitre de cette partie. Dans ce processus de réflexion, de décision et de mise en œuvre, du temps et de l'énergie doivent être consacrés à chacune des étapes. L'entrepreneur n'a pas le temps de tirer au clair tout ce qui lui arrive : il est pris dans un tourbillon, un feu roulant. Puis, tout à coup, tout s'arrête. Les émotions peuvent refaire surface. Le détachement face à la situation devient alors nécessaire.

L'ouvrage renseigne sur les différentes phases que l'entrepreneur risque de traverser et sur les références utiles afin d'apporter du soutien en cas de besoin. Le lecteur est aidé dans la compréhension de ses émotions. Toutefois, selon moi, le message le plus important est le fait que « seul le temps » pourra venir à bout des sentiments négatifs pouvant se dégager de toute la démarche.

\section{$4^{\mathrm{e}}$ partie : rebondir}

La dernière partie contient un seul chapitre. L'acte de rebondir peut se faire de différentes façons, mais les autrices l'abordent avec un souci particulier : l'entrepreneur doit rester actif et prendre soin de sa santé mentale et physique. Ainsi, après la réflexion, le choix et la mise en œuvre, qui impliquent un temps d'arrêt dans le but de digérer toutes les étapes précédentes, arrive le temps d'entrevoir le futur.

«Que veux-tu faire quand tu seras grand? » En principe, à cette étape, l'entrepreneur est à la phase de reconstruction. Il a su passer à travers toute la gamme d'émotions, il est bien reposé et est prêt pour un autre défi. Peut-être est-il suffisamment «grand» pour considérer que le temps restant sera consacré à profiter de la vie avec sa famille? Peut-être est-il un hyperactif et aura-t-il envie de relever de nouveaux défis? Peut-être considère-t-il même se lancer de nouveau en affaires? Même s'il a vécu un échec, il peut être prêt à se remettre en selle afin de mettre à profit les nouvelles connaissances acquises au cours de cette épreuve. Ou, au contraire, il a eu sa leçon et n'a plus d'intérêt pour ce type d'aventure. Il préfère alors s'orienter vers un emploi qui le valorisera, en plus de l'aider à gagner sa vie. 
Bref, il y a autant de situations que d'entrepreneurs. Sa santé, physique, mentale et financière aura un impact dans le succès de cette phase finale.

\section{Conclusion}

J'ai bien aimé une phrase dans la conclusion. À mon avis, elle résume bien ce que je pense du sujet : «N'oubliez jamais que toute fin est le commencement de quelque chose ». Je crois que cette phrase résume aussi la nature de l'ouvrage. Il sert à vulgariser une étape inévitable dans la vie d'un entrepreneur : sa sortie.

Le livre a un côté très pratique. En raison de ma formation et de mes expériences, je comprenais mieux certains éléments présentés, soit parce que je les ai vécus, soit parce que je les ai étudiés. Par contre, pour l'entrepreneur n'ayant pas une formation dans le domaine de l'administration, et plus spécifiquement en comptabilité, il s'agira d'un ouvrage très utile. Il démystifie plusieurs aspects du processus de sortie, qu'il soit volontaire ou non.

Selon moi, il constitue un livre de chevet, un journal personnel qui accompagne le lecteur de façon individuelle dans ce qu'il vit. Il y a quelque chose de rassurant dans les exemples donnés qui fait en sorte qu'il se sentira moins seul, tout en étant seul, ce qui est paradoxal. En résumé, même si chaque contexte de sortie d'un entrepreneur est différent, l'ouvrage fournit un bon guide général sur la façon de l'aborder. 